\title{
Structure-Based Optimization of a Potent PACE4 Inhibitor Containing a Decarboxylated P1 Arginine Mimetic
}

\author{
Anna Kwiatkowska, Christine Levesque, Frédéric Couture, Kévin Ly, \\ Frédérik Dufour, and Robert Day
}

Institut de pharmacologie de Sherbrooke (IPS) et département de chirurgie/service d'urologie, Faculté de médecine et des sciences de la santé (FMSS), Université de Sherbrooke, Sherbrooke, J1H 5N4, Québec, Canada

\section{Introduction}

Our recent studies have provided direct evidence for the critical role of PACE4 in the progression of prostrate cancer, identifying this enzyme as a promising target to design novel and effective treatments [1]. Moreover, we developed a potent PACE4 inhibitor with considerable selectivity (20-fold over furin) known as the Multi-Leu (ML) peptide [2]. In order to improve its pharmacological profile, we performed structure-activity relationship (SAR) studies and determined that the incorporation of the decarboxylated arginine mimetic (4-amidinobenzylamide, Amba) at the P1 position led to a more potent and stable analog [3]. Unfortunately, this inhibitor suffered from a reduced selectivity towards PACE4. To restore its specificity profile, we used a positional-scanning approach and synthesized peptide libraries by substituting each amino acid residue in the leucine core of our inhibitor. These studies revealed that we are able to enhance the specificity profile (3-fold) and preserve the inhibitory activity as well as antiproliferative properties of our inhibitor by incorporating a leucine isomer L-isoleucine into its structure (Maluch, et al., unpublished data). Based on these results, we decided to perform further SAR studies aiming to improve the specificity and activity of our MLAmba inhibitor. We focused on the leucine core (P8-P5) and its modification with unnatural amino acid residues possessing hydrophobic character (Figure 1). First we evaluated the impact of a single substitution (from the P8 to P5 position) on the inhibitory activity of the resulting peptides, and then we combined the most promising modifications. In this work, we present the synthesis and biological evaluation of a new series of MLAmba analogs.

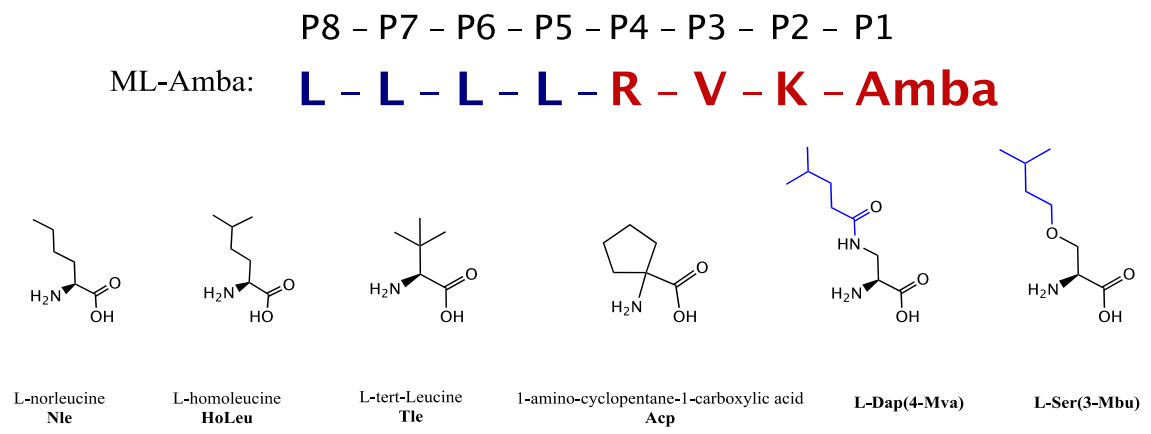

Fig. 1. The amino acid residues selected to modify the leucine core (P8-P5 position) of the MLAmba inhibitor.

\section{Results and Discussion}

All peptides were obtained by a combination of solid-phase peptide synthesis and solution synthesis as previously described [3]. The scan of the P5, P6 and P7 (with the exception of the Tle ${ }^{\mathrm{P} 7}$ ) position resulted in analogs with reduced inhibitory potency against PACE4 when compared to the initial peptide (MLAmba), revealing that Leu residues at these positions provide higher binding affinity for the enzyme (Figure 2A). On the other hand, the Tle-substitution at the P7 and P8 positions had a beneficial effect and led to peptides with improved activity, while the Acp ${ }^{\mathrm{P} 8}$-analog maintained potency similar to the MLAmba inhibitor. In the case of analogs with combined modifications, only 
one peptide substituted at the P8 and P5 positions with the Nle displayed enhanced activity against PACE4 (Figure 2B).

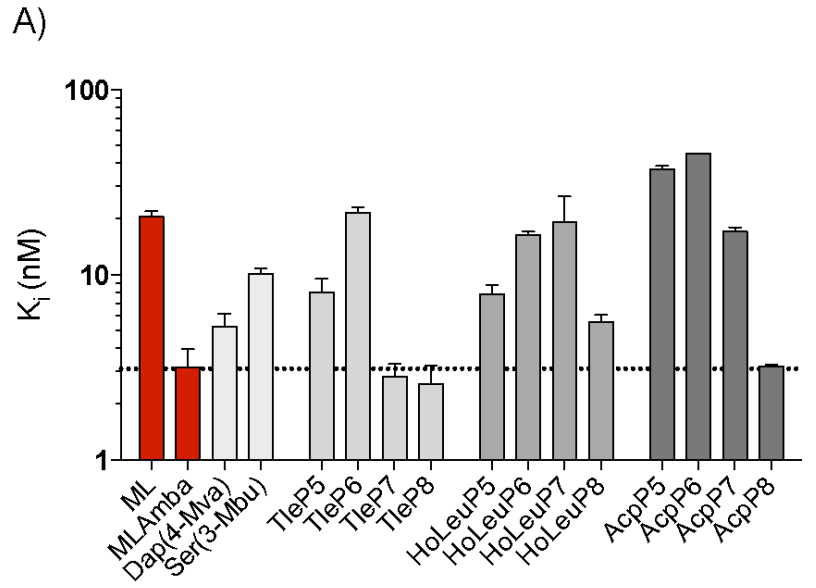

B)

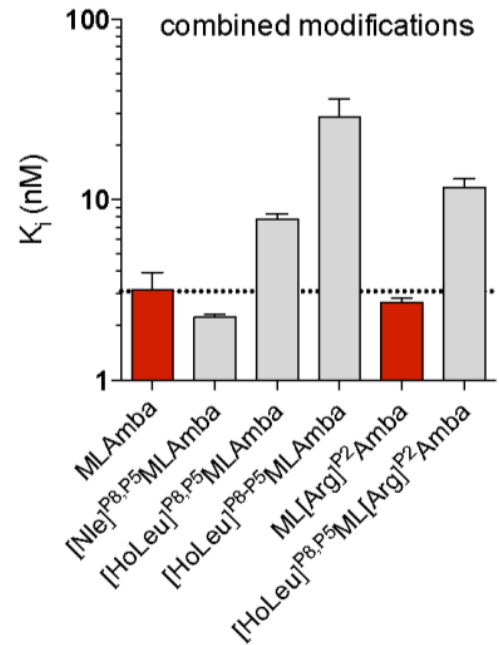

Fig. 2. Inhibitory activity of the new MLAmba analogs towards PACE4. A) Positional scanning in the leucine core of the MLAmba peptide. B) Activity of the analogs containing multiple substitutions. Results are means $\pm S D$ from three independent experiments. Control peptides are indicated in red.

Regarding the antiproliferative activity, the incorporation of the Tle residue to the MLAmba peptide yielded analogs with significantly diminished potency, whereas the HoLeu-substitution preserved the inhibitory activity of the resulting peptides (Table 1). In the case of the Ser(3-Mbu) ${ }^{\mathrm{P} 5}$-modified peptide, its activity towards DU145 and LNCaP cells was reduced (3.2- and 4.2-fold, respectively), while the compound containing $\mathrm{Acp}^{\mathrm{P} 8}$ or $\operatorname{Dap}(4-\mathrm{Mva})^{\mathrm{P} 8}$ showed no inhibitory effect.

Table 1. Antiproliferative activity and cytotoxicity of the selected analogs.

\begin{tabular}{|c|c|c|c|}
\hline Analog & $\begin{array}{c}I C_{50}(\mu M) \\
\pm S D \\
(D U 145) \\
\end{array}$ & $\begin{array}{c}I C_{50}(\mu M) \\
\pm S D \\
(L N C a P) \\
\end{array}$ & $\begin{array}{c}L D H(D U 145) \\
\text { at IC } C_{50} \\
\text { concentration }\end{array}$ \\
\hline Ac-LLLLRVK[Amba] & $25 \pm 10$ & $40 \pm 15$ & $<5 \%$ \\
\hline$[\mathrm{Tle}]^{\mathrm{P} 5}$ MLAmba & $115 \pm 10$ & $180 \pm 50$ & $<5 \%$ \\
\hline$[\text { Tle }]^{\mathrm{P} 6}$ MLAmba & $150 \pm 25$ & N.C. & $<5 \%$ \\
\hline$[\mathrm{Tle}]^{\mathrm{P7}}$ MLAmba & $120 \pm 5$ & N.C. & $<5 \%$ \\
\hline$[\text { Tle }]^{\mathrm{P} 8}$ MLAmba & $130 \pm 10$ & N.C. & $<5 \%$ \\
\hline$[\text { HoLeu }]^{\mathrm{P5}}$ MLAmba & $30 \pm 5$ & $50 \pm 10$ & $<5 \%$ \\
\hline$[\text { HoLeu }]^{\mathrm{P} 6}$ MLAmba & $40 \pm 10$ & $40 \pm 10$ & $<5 \%$ \\
\hline$[\text { HoLeu }]^{\mathrm{P7}}$ MLAmba & $30 \pm 5$ & $80 \pm 25$ & $<5 \%$ \\
\hline$\left[\right.$ HoLeu ${ }^{\mathrm{P} 8}$ MLAmba & $30 \pm 10$ & $30 \pm 10$ & $<5 \%$ \\
\hline$[\mathrm{Acp}]^{\mathrm{P} 8}$ MLAmba & N.C. & N.C. & $<5 \%$ \\
\hline$[\mathrm{Dap}(4-\mathrm{Mva})]^{\mathrm{P} 8}$ MLAmba & N.C. & N.C. & $<5 \%$ \\
\hline$[\operatorname{Ser}(3-\mathrm{Mbu})]^{\mathrm{P5}} \mathrm{MLAmba}$ & $80 \pm 5$ & $170 \pm 20$ & $<5 \%$ \\
\hline$[\mathrm{Nle}]^{\mathrm{P} 8, \mathrm{P} 5 \mathrm{MLAmba}}$ & $50 \pm 10$ & $85 \pm 10$ & $<5 \%$ \\
\hline$[\text { HoLeu }]^{\mathrm{P} 8, \mathrm{P} 5}$ MLAmba & $15 \pm 1$ & $30 \pm 3$ & $<5 \%$ \\
\hline$[\text { HoLeu }]^{\mathrm{P}-\mathrm{P} 5}$ MLAmba & $11 \pm 1$ & $130 \pm 70$ & $<5 \%$ \\
\hline
\end{tabular}

IC 50 s were calculated from the dose-response curves using DU145 and LNCaP cell lines. The lactate dehydrogenase $(L D H)$ release (an indicator of acute cytotoxicity) was quantified relative to the untreated control. Results are means $\pm S D$ from three independent experiments, N.C. - not calculable. 
The modification of the MLAmba peptide with Nle residues at the P5 and P8 position slightly reduced its antiproliferative activity, whereas the incorporation of multiple HoLeu substitutions greatly improved it. However, it was shown that some of the most potent analogs in this test exhibited not only an antiproliferative effect on cancer cells, but were also cytotoxic at higher concentration (Figure 3).

Regarding the selectivity profile of the new MLAmba analogs over furin, in general the selected modifications did not result in peptides with improved selectivity with the exception of two compounds substituted with $\mathrm{HoLeu}^{\mathrm{P} 5}$ and $\mathrm{Acp}^{\mathrm{P} 8}$, which were more selective towards PACE4 (2- and 3-fold respectively) when compared to the initial peptide.

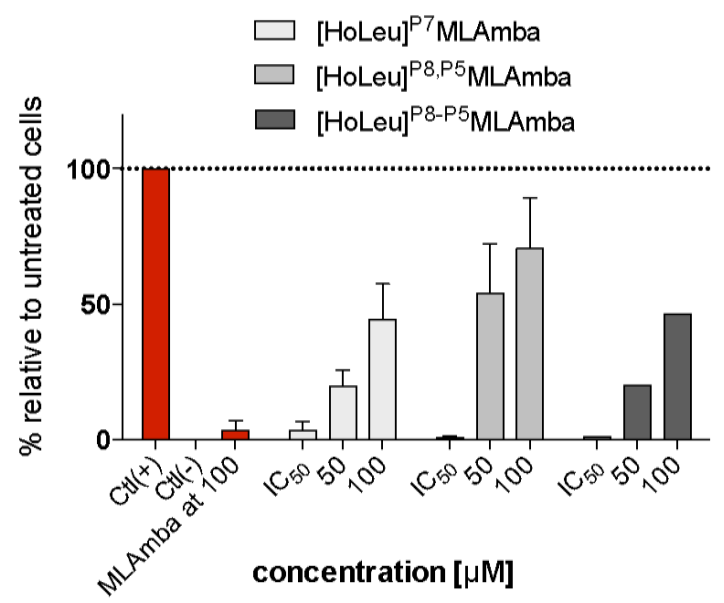

Fig. 3. Cytotoxic effect ( $L D H$ release) of the selected analogs at various concentrations on DU145 cells.
These data demonstrate that the modification of the leucine core of the MLAmba inhibitor has crucial impact on the inhibitory activity of the resulting peptides. Among all the modification tested, the HoLeu residue turned out to be the most favorable. Although, HoLeu-substituted analogs did not exhibit enhanced potency in the kinetic assay with recombinant PACE4, they showed comparable or improved antiproliferative activity against prostate cancer cells. Bearing in mind that the incorporation of the unnatural amino acids into a peptide sequence should also improve its stability, we are undertaking further studies on our analogs and we will test their stability in plasma. We believe that these findings lay the foundation for future research focusing on the development of more potent, specific and stable PACE4 inhibitors with the potential to become new drug candidates to treat prostate cancer.

\section{Acknowledgments}

The authors thank Xue Wen Yuan for assistance with peptide synthesis, Roxane Desjardins for enzyme preparation, Sophie Beauchemin for the synthesis of Fmoc-L-Ser(3-Mbu) and assistance with the synthesis of the Amba modification, and Nicolas Dory for help with enzyme kinetic studies. AK holds a Heart and Stroke Foundation postdoctoral fellowship. FC holds a Banting and Charles Best Canada Graduate Scholarships (Grant \#315690) from CIHR and a Graduate Studentship from Prostate Cancer Canada (Grant \#GS-2015-07). FD holds a MS Student Scholarships from the FRQ-S and CIHR.

\section{References}

1. Couture, F., et al. Neoplasia 14, 1032-1042 (2012). http://dx.doi.org/10.1593/neo.121368

2. Levesque, C., et al. J. Med. Chem. 55, 10501-10511(2012), http://dx.doi.org/10.1021/im3011178

3. Kwiatkowska, A., et al. J. Med. Chem. 57, 98-109 (2014), http://dx.doi.org/10.1021/jm401457n 